OPEN

SUBJECT AREAS:

NEURODEVELOPMENTAL

DISORDERS

CELLULAR NEUROSCIENCE

PAEDIATRIC NEUROLOGICAL

DISORDERS

Received

9 July 2014

Accepted

3 December 2014

Published

15 January 2015

Correspondence and requests for materials should be addressed to

J.G.S. (joachim. schulz@uk-essen.de) or C.G.D. (cdotti@cbm. csic.es)

\section{Glial $\beta$-Oxidation regulates Drosophila Energy Metabolism}

\author{
Joachim G. Schulz' , Antonio Laranjeira' , Leen Van Huffel' , Annette Gärtner' , Sven Vilain' , Jarl Bastianen', \\ Paul P. Van Veldhoven ${ }^{2} \&$ Carlos G. Dotti ${ }^{1,3}$
}

${ }^{1}$ VIB Center for the Biology of Disease, Leuven and Center for Human Genetics, KU Leuven, Leuven, Belgium, ${ }^{2}$ Laboratory of Lipid Biochemistry, KU Leuven, Herestraat 49, 3000 Leuven, Belgium, ${ }^{3}$ Centro Biología Molecular "Severo Ochoa" CSIC-UAM, Madrid, Spain.

The brain's impotence to utilize long-chain fatty acids as fuel, one of the dogmas in neuroscience, is surprising, since the nervous system is the tissue most energy consuming and most vulnerable to a lack of energy. Challenging this view, we here show in vivo that loss of the Drosophila carnitine

palmitoyltransferase 2 (CPT2), an enzyme required for mitochondrial $\beta$-oxidation of long-chain fatty acids as substrates for energy production, results in the accumulation of triacylglyceride-filled lipid droplets in adult Drosophila brain but not in obesity. CPT2 rescue in glial cells alone is sufficient to restore triacylglyceride homeostasis, and we suggest that this is mediated by the release of ketone bodies from the rescued glial cells. These results demonstrate that the adult brain is able to catabolize fatty acids for cellular energy production.

r o guarantee organismal survival, a continuous supply of ATP for cellular energy production is maintained by many regulatory mechanisms at the cellular, endocrine and behavioral level. Under normal feeding conditions, ATP is generated from different substrates, such as carbohydrates, amino and fatty acids, delivered to the various tissues via the circulation. When calories are oversupplied, triacylglycerides (TAG) are synthesized from the different substrates and stored in specific cells, so called adipocytes. Excess body TAG storage is called obesity, a condition that is linked to increased morbidity and mortality. During prolonged starvation, cellular TAG mobilization and catabolism becomes vital. The long-chain fatty acid (LCFA) groups are released from the glycerol backbone and activated with a Coenzyme A (CoA) residue. LCFA-CoA is catabolized through $\beta-$ oxidation, either in mitochondria or in peroxisomes, but only mitochondrial $\beta$-oxidation is coupled to ATP production $^{1}$. LCFA-CoA does not cross the tight inner mitochondrial membrane: to cross, the CoA residue is replaced with a carnitine residue, a reaction that is catalyzed by carnitine palmitoyltransferase 1 (CPT1). LCFA-carnitine is transported with carnitine-acylcarnitine translocase (CACT) across the inner membrane. Inside mitochondria, carnitine needs to be replaced again by CoA, a reaction catalyzed by carnitine palmitoyltransferase 2 (CPT2), yielding LFCA-CoA that can be catabolized through $\beta$-oxidation and generate ATP $^{2-4}$.

Human patients with severe mutations in one of the enzymes of the carnitine shuttle system, CPT1 or CPT2, die early after birth. Patients with less severe mutations are sensitive to starvation throughout life. This is probably a direct consequence of the failure to generate ATP through the TAG- $\beta$ - oxidation pathway ${ }^{5,6}$ and illustrates the critical importance of this pathway.

Although CPT2 deficiency patients have brain defects ${ }^{7,8}$, and CPT1 and CPT2 expression has been demonstrated in adult brain ${ }^{5,9}$, it has been stated that the brain does not use $\beta$-oxidation for energy production ${ }^{10}$. In fact, under conditions of starvation brain metabolism is thought to be restricted to use ketone bodies derived from $\beta$ oxidation of long-chain fatty acids in the liver. It has been argued that the reason behind this might be that $\beta$ - oxidation $^{11}$ is associated with the production of free oxygen radicals, causing neuronal loss. While liver or muscle cells, where $\beta$-oxidation occurs at high rates during starvation, are replaceable, brain cells are postmitotic, unique in terms of circuitry and therefore not easily, if at all, replaceable. Another reason for the denial of $\beta$-oxidation in the brain may reside in the limited space in the scull or head capsule to allow for extensive lipid storage without compromising brain function.

To challenge the view that the brain does not use long chain fatty acids for energy production, we here directly tested the contribution of the brain in organismal $\beta$-oxidation by removing dCPT2 in Drosophila melanogaster. Our results show that $\beta$-oxidation does in fact occur in the glial cells of the adult Drosophila brain in vivo and may contribute to organismal energy supply. 


\section{Results}

Drosophila CPT2 deficiency resembles human CPT2 deficiency. To determine the importance of fatty acid catabolism for energy production in brain, we searched the Drosophila melanogaster genome for the orthologue of the highly conserved bottleneck enzyme of $\beta$-oxidation, carnitine palmitoyltransferase 2 (CPT2).

Based on BLAST search on the Drosophila genome, we identified $C G 2107$, from now on called $d C P T 2$ (Fig. 1a), on chromosome 3L, and generated loss-of function alleles by imprecise excision of P-element $P(G S V 7) G S 21694$ situated in the $5^{\prime}$ UTR of the only $d C P T 2$ transcript, 92 base pairs before the translational start site. Two mutant alleles, $d C P T 2^{\delta 3}$ and $d C P T 2^{\delta 18}$, we considered null mutants, since a major part of the ORF (508 and 124 of the 667 amino acids, respectively) was deleted (Fig. 1b), and both mutants failed to complement each other, or Deficiencies $D f(3 L) B S C 428$ and $D f(3 L) B S C 671$ spanning the CG2107 locus (Fig. 1c). As wild type control line in our experiments we used the revertant line giving rise to a precise excision of $P(G S V 7) G S 21694$.

The majority (62\%) of the $d C P T 2^{\delta 3}$ mutant flies died within the first three days after eclosion, reminiscent of the postnatal lethality in severe human CPT2 deficiency patients. This phenotype was fully rescued with ubiquitous expression of wild type rat CPT2 (rCPT2) (p $=0.0003)$, but not with inactive rat CPT2 bearing the H372A mutation of the catalytic site that gives rise to human disease ${ }^{12}$ (Fig. 1c). This confirms that CG2107 is indeed orthologous to mammalian CPT2, and that $d C P T 2^{\delta 3}$, from now on called $d C P T 2^{-/-}$, specifically affects $d C P T 2$. The ectopic expression of rat CPT2 in flies was confirmed by Western blot (Fig. 1d).

dCPT2 is required in adult life. Developmental analysis showed that most of the $d C P T 2^{-1-}$ lethality occurred in the first three days after eclosion $(\mathrm{p}=0.003)$, and very little during larval or pupal development (Fig. 2a). $d C P T 2^{-/-}$flies that survived the immediate post-eclosion period still showed a severely reduced adult lifespan of 22 and 10 days in females and males, respectively, compared to 47 and 42 days in control females and males (Fig. 2b). The reduced lifespan could be fully reversed by ubiquitous rescue of CPT2. This indicates that flies require $\beta$-oxidation for survival during adulthood.

Another core symptom of human CPT2 deficiency in adults is sensitivity to starvation. Similar to human CPT2 deficiency patients, $d C P T 2^{-\prime-}$ flies were hypersensitive to starvation. Under conditions of complete starvation, the average survival time of $d C P T 2^{-1-}$ flies dropped from $63.1+/-7.5$ hours to $37.5+/-2.6$ hours in female $(\mathrm{p}=0.005)$ and from $32.0+/-3.4$ to $21.3+/-3.3$ hours in male $(\mathrm{p}$ $=0.017) d C P T 2^{-1-}$ flies when compared to wild type (Fig. 2c).

No obesity but brain TAG accumulation in adult $d C P T 2^{-/-}$flies. We asked if systemic lack of mitochondrial $\beta$-oxidation causes obesity, since it is the main pathway for long-chain fatty acid (LCFA) degradation. In contrast to our expectations, normally fed $d C P T 2^{-1-}$ flies were not heavier (Fig. 2d) but even lighter than controls, $0.72+/-0.04 \mathrm{mg}$ vs. $0.67+/-0.04 \mathrm{mg}$ for male $(\mathrm{p}=$ $0.02)$ and $1.04+/-0.05 \mathrm{mg}$ vs. $0.97+/-0.02 \mathrm{mg}$ for female $(\mathrm{p}=$ 0.002 ) wild type vs. $d C P T 2^{-\prime-}$ flies, respectively. Body length was also somewhat reduced in $d C P T 2^{-1-}$ flies when compared to controls $(2.70+/-0.13$ vs. $2.61+/-0.08 \mathrm{~mm}$ in female $(\mathrm{p}=0.02)$ and $2.25+/-0.11 \mathrm{~mm}$ vs. $2.23+/-0.16 \mathrm{~mm}$ in male (not significant) wild type vs. $d C P T 2^{-1-}$ flies, respectively (Fig. 2e), but the ratio of weight and size (BMI) was not increased in dCPT2-/ - flies (Fig. 2f). Motor activity over a period of $24 \mathrm{~h}$ was also unchanged, reaching from $469+/-63$ beam passes in control to $535+/-70$ in heterozygotes to $538+/-80$ in $d C P T 2^{-/-}$homozygotes (Fig. $2 \mathrm{~g}$ ), ruling out increased muscle work as the cause for the lack of an obesity phenotype in $d C P T 2^{-\prime-}$ animals. Together this suggests that in Drosophila the regulation of energy balance is independent from mitochondrial $\beta$-oxidation.
Whole body neutral lipid was not increased in fed $d C P T 2^{-1-}$ flies. In fed male $d C P T 2^{-1-}$ flies, TAG levels were identical in both $d C P T 2^{-1-}$ and wild type flies. In fed female $d C P T 2^{-/-}$flies, TAG levels were even lower than in wild type controls (Fig. 3a). Total TAG was turned over at the same rate in $d C P T 2^{-/-}$and wild type flies under starvation conditions (Fig. 3a). This result further strengthens the notion that pathways other than mitochondrial $\beta$ oxidation regulate TAG turnover at the organismal level.

Yet, although TAG levels were not increased in the body of $d C P T 2^{-1-}$ flies, TAG accumulated massively in $d C P T 2^{-1-}$ brains. Consistent with what is known from mammalian brains, in wild type brains TAG was barely detectable (Fig. 3b, c). TAG levels in wild type fly brain were at the detection limit of 13.4 pmol per brain, while reaching 235.1 pmol TAG per $d C P T 2^{-1-}$ brain, equivalent to a 17.5 fold increase $(p=0.0004)$. Brain phospholipid levels were unchanged, ruling out a simple increase in brain size, brain degeneration or loss of brain tissue due to the lack of dCPT2.

In order to validate and further understand the nature of the TAG accumulations in $d C P T 2^{-1-}$ brains, we performed brain whole mount staining with a neutral lipid specific dye ${ }^{13}$. This confirmed widespread accumulation of neutral lipid droplets in $d C P T 2^{-1-}$ brains, while basically no signal was obtained from wild type brains (Fig. 3d, middle panel). Similar results were obtained from fly brains mutant for $d C P T 1$ (Fig. 3d, right panel), another bottle-neck enzyme required for mitochondrial $\beta$-oxidation, giving rise to a similar human phenotype. Interestingly, lipid droplets also accumulated in flight muscle (Fig. 3d).

Finally, electron microscopy images from $d C P T 2^{-1-}$ but not control brains showed frequent droplet-like inclusions of homogenous appearance, a single outer membrane, and variable size, characteristic for TAG containing lipid droplets (Fig. 3e).

Altogether, the last series of results demonstrates massive accumulation of TAG-filled lipid droplets in the brains and muscle of $d C P T 2^{-1-}$ animals while total body TAG levels or body were not increased in $d C P T 2^{-1-}$ animals.

Glial $\beta$-oxidation restores lipid homeostasis. We analyzed next if $\mathrm{dCPT} 2$ is expressed in the adult fly brain. Using a knock in line where GFP is fused to dCPT2 in the $d C P T 2$ locus, we could demonstrate endogenous expression of dCPT2 in the brain by confocal microscopy (Supplementary data). Knockdown of dCPT2 by RNA interference specifically in neurons or glia caused a decrease of $d C P T 2$ transcript levels to $67 \%$ and $28 \%$ of control levels in brain, respectively (Fig. 4a). Considering that in Drosophila the large majority of brain cells are neurons this suggests that in adult fly brain dCPT2 is expressed predominantly in glia and the brain TAG deposits in $d C P T 2^{--}$flies are located in glial cells.

To prove this point, we ectopically expressed lipid storage droplet2 (LSD-2), a molecular marker for lipid droplets ${ }^{14}$ specifically in neurons or glia by driving UAS-LSD-2 with Nsyb-Gal4 or RepoGal4, respectively, and analyzed the brains of adult flies by confocal microscopy. We found co-localization of LSD-2 with TAG deposits in glia but not neurons of $d C P T 2^{-/-}$flies (Fig. $4 \mathrm{~b}$ ), demonstrating stabilization of LSD-2 in lipid droplets in glia but not in neurons.

Is glial $\beta$-oxidation functionally relevant? To answer this, we performed glial-specific dCPT2 rescue experiments. Glial-specific rescue of $d C P T 2^{-1-}$ flies reversed brain TAG accumulation (Fig. 4c) and peri-eclosion lethality $(\mathrm{p}=0.0009)$ (Fig. $4 \mathrm{~d})$, and normalized lifespan partially (Fig. 4e).

To specify further the role of dCPT2 in glial cells, we performed rescue experiments in subpopulations of glial cells of $d C P T 2^{-/-}$ flies ${ }^{15,16}$. This revealed that dCPT2 expression in cortex glia (NP2222-Gal4; $p=0.004$ and Nrv2-Gal4; $p=0.0009$ ) or astrocyte-like glia (dEAAT1-Gal4; $\mathrm{p}=0.0005$ ) but not in ensheathing glia (NP6520) is sufficient to restore eclosion of $d C P T 2$ mutants (Fig. 4f). 


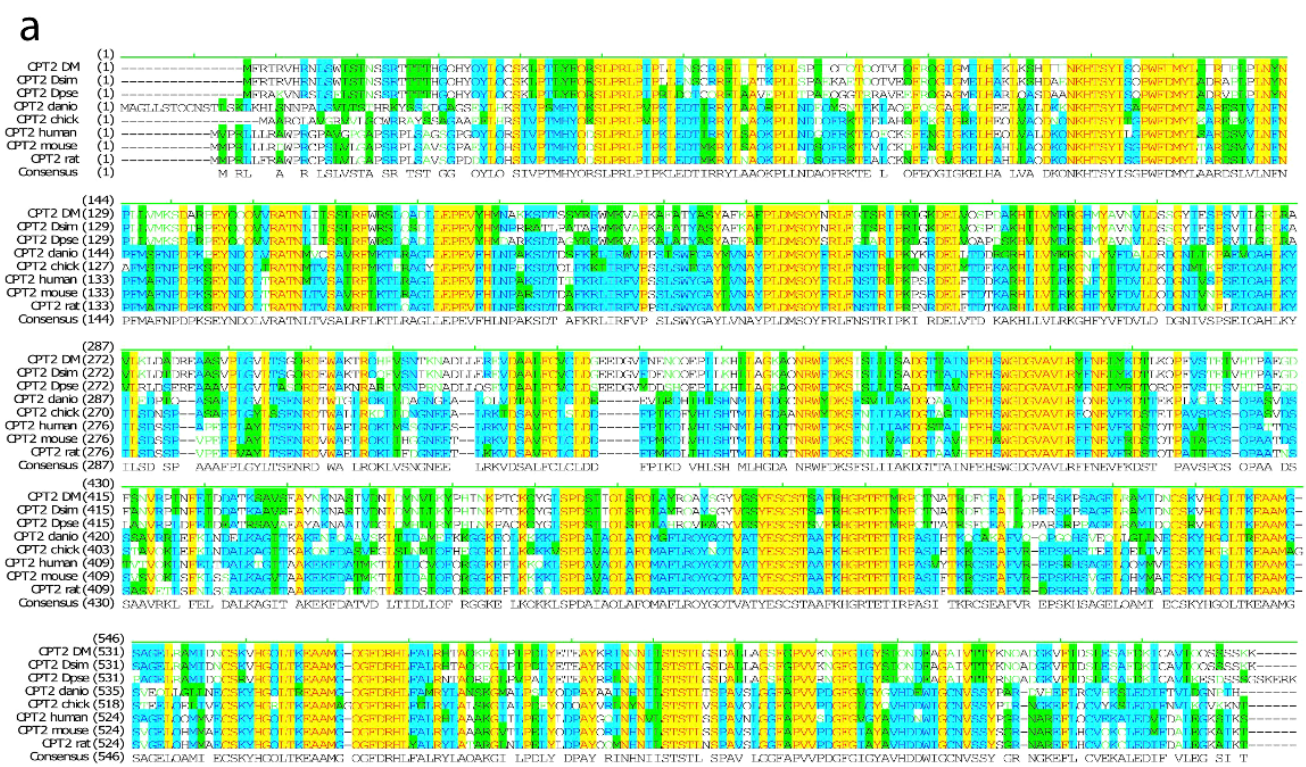

b

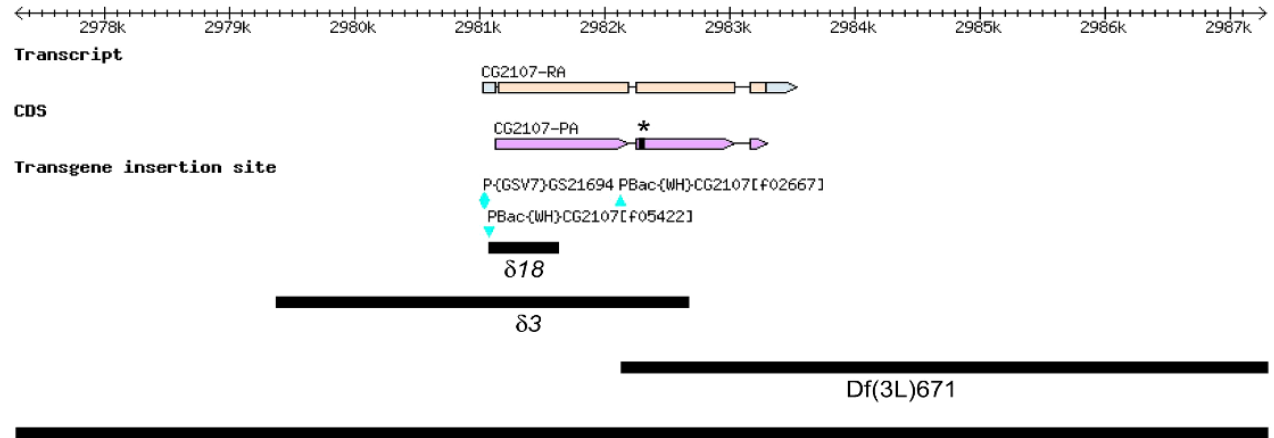

$\operatorname{Df}(3 L) 428$
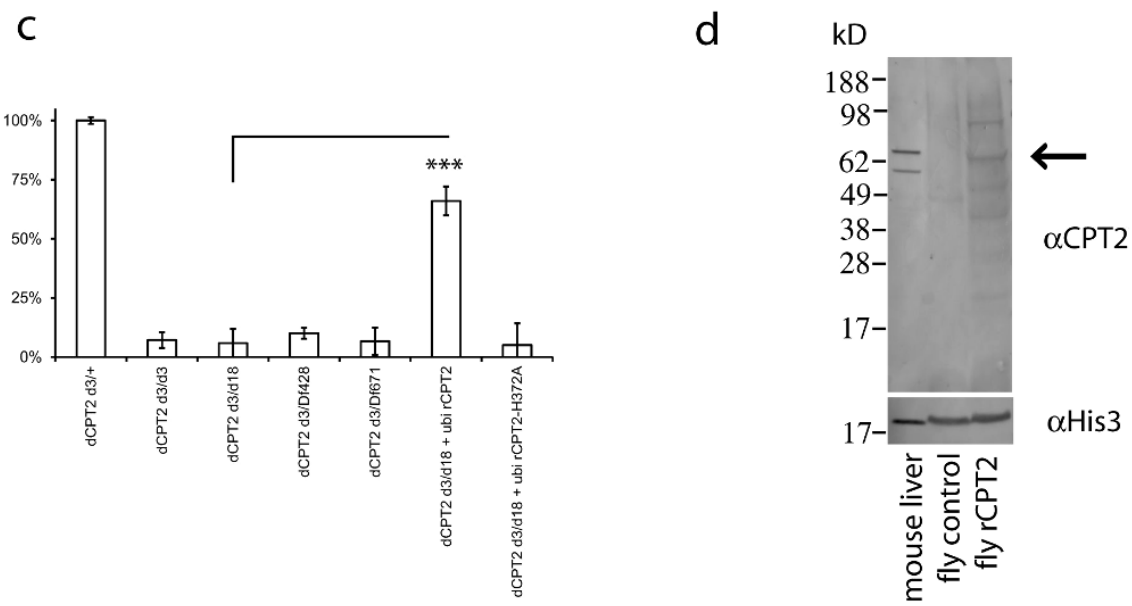

Figure 1 | CG2107 mutants and rescue with mammalian CPT2. (A) Protein sequence alignment of Drosophila melanogaster (DM), Drosophila simulans (Dsim), Drosophila pseudoobscura (Dpse), Danio rerio (danio), gallus gallus (chick), homo sapiens (human), mus musculus (mouse), rattus norvegicus (rat) and consensus CPT2. (B) Region 63A2 of Drosophila melanogaster chromosome 3L, bearing dCPT2 (CG2107), P-element insertions, deficiencies $\operatorname{Df}(31) 428$ and Df(3L)671 spanning the CG2107 locus, and the sequences deleted in two newly generated mutants $d C P T 2^{\delta 18}$ and ${ }^{83}$. (C) Eclosion rates of dCPT2 mutant flies with the indicated genotypes in percent of expected ( $\mathrm{n}>=3$ experiments, ${ }^{* * * *} \mathrm{p}<0.0001$ ). Rescue was performed with wild type (rCPT2) or mutant $\left(\right.$ rCPT2 ${ }^{\mathrm{H} 372 \mathrm{~A}}$ ) rat CPT driven by the ubiquitous driver da-Gal4. (D) Western blot from mouse liver or whole fly extract from wild type or rCPT2 expressing flies, stained with anti-CPT2 to confirm ectopic expression in rescue flies ( $65 \mathrm{kDa}$ band marked with an arrow), or anti-Histone 3 as loading control. 

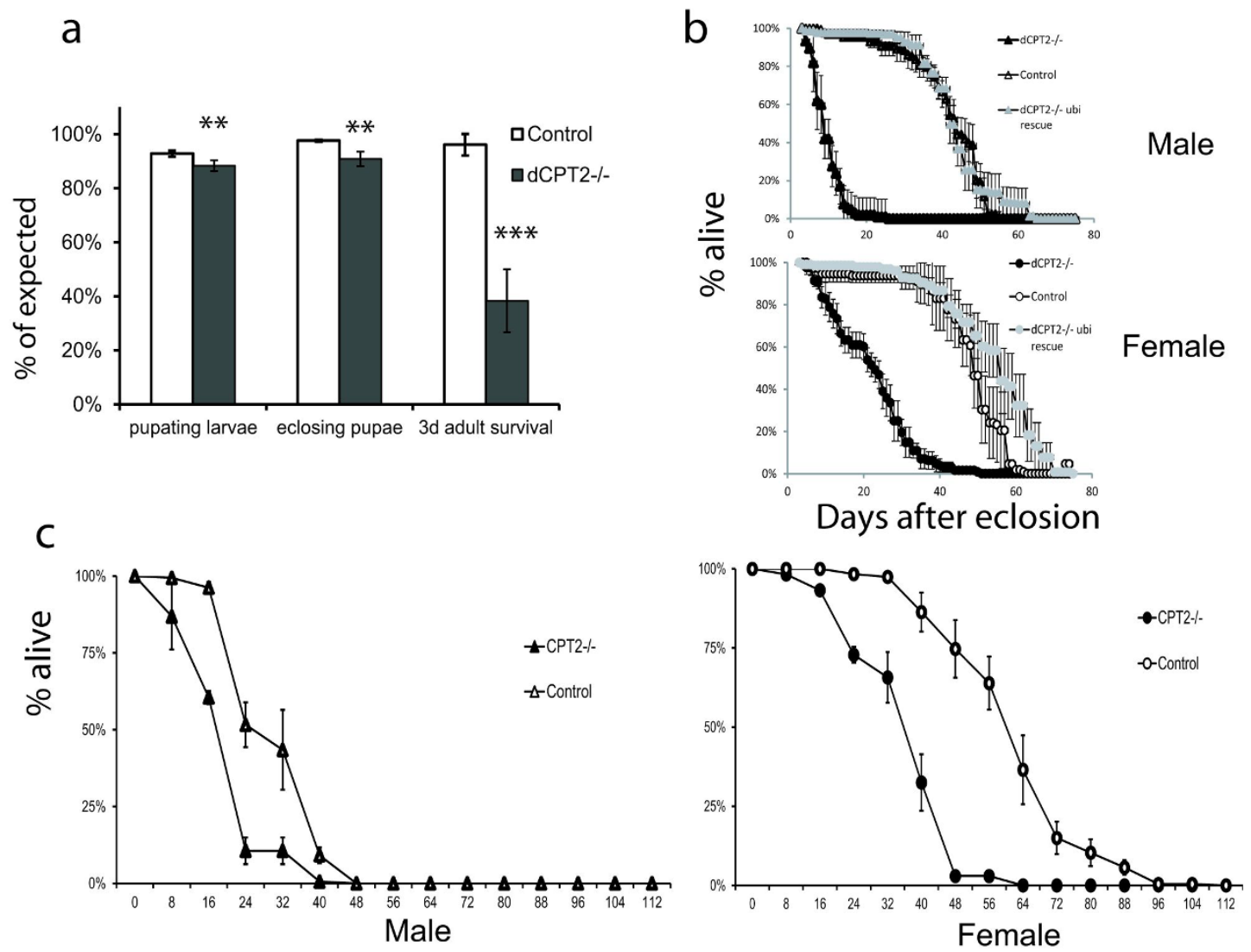

Hours of starvation

d
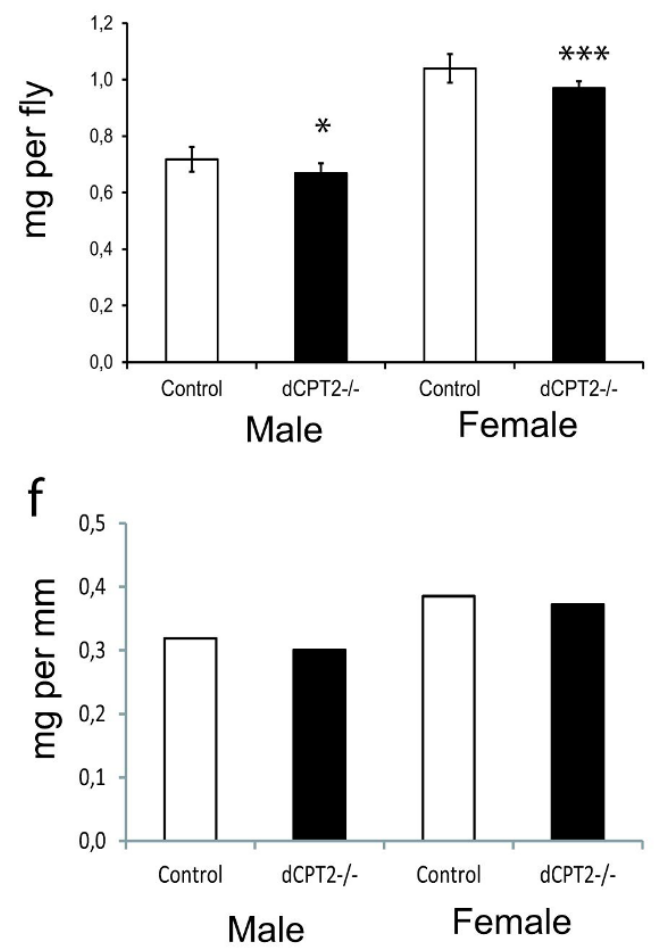

e

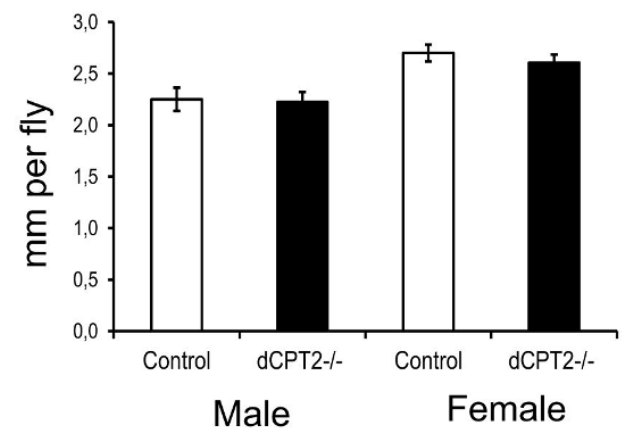

9

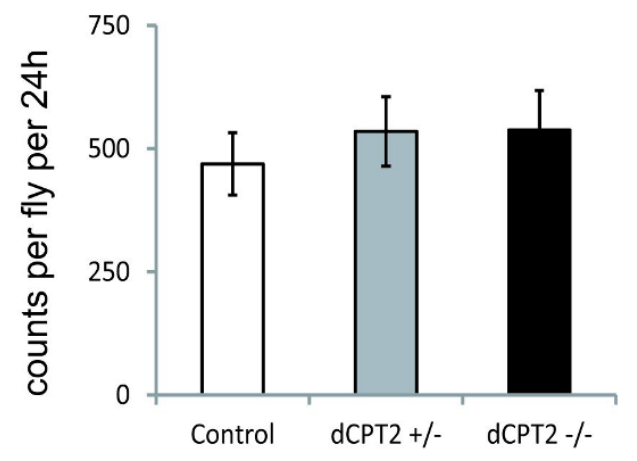

Figure $2 \mid$ Phenotype of $d C P T 2^{-/-}$animals. (A) Percent survival of wild type (Rev) or $d C P T 2^{-/-}$larvae, pupae or young adults, in percent (n $>=3$ experiments; ${ }^{* *} \mathrm{p}<0.01 ; * * \mathrm{p}<0.005$ ). (B) Lifespan of wild type or $d C P T 2^{-/-}$adults with or without ubiquitous rescue with rCPT2, in days $+/-$ standard error of the means ( $\mathrm{n}>=4$ experiment for a total of $>=105$ animals per genotype). (C) Percent survival of starving 1 week old adults on $1 \%$ agarose, in hours $+1-$ standard error of the means $(\mathrm{n}=3$ for a total of $>=209$ animals per genotype). (D) Weight per adult fly, in $\mathrm{mg}(\mathrm{n}>=394$; * $\mathrm{p}<$ $0.05 ; * * * p 0.005)$ ). (E) Length of adult flies, in $\mathrm{mm}(\mathrm{n}=10)$. (F) Ratio of weight per length in $\mathrm{mg} / \mathrm{mm}$. (G) Total motor activity time of adult male flies, in counts per $24 \mathrm{~h}(\mathrm{n}=6)$. 
a
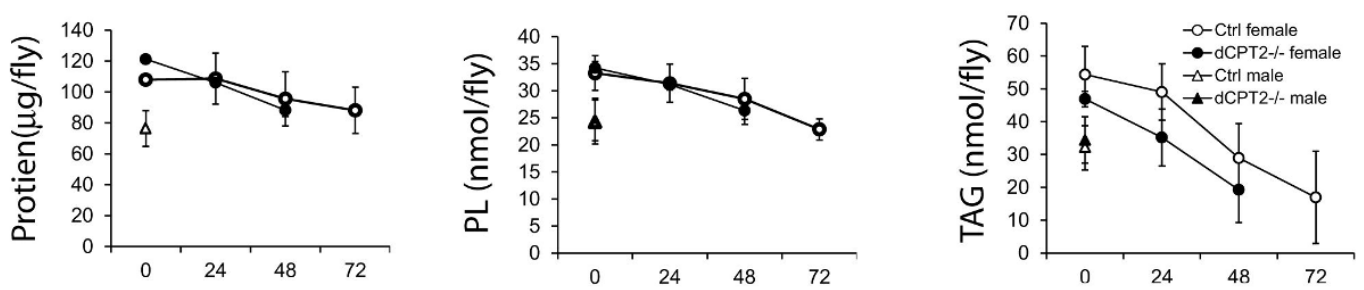

b
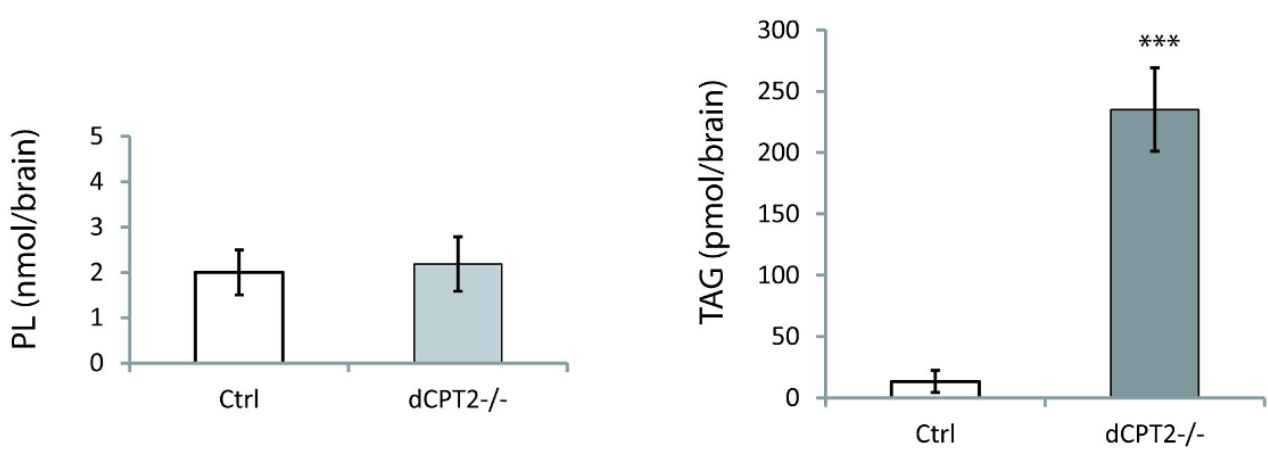

C

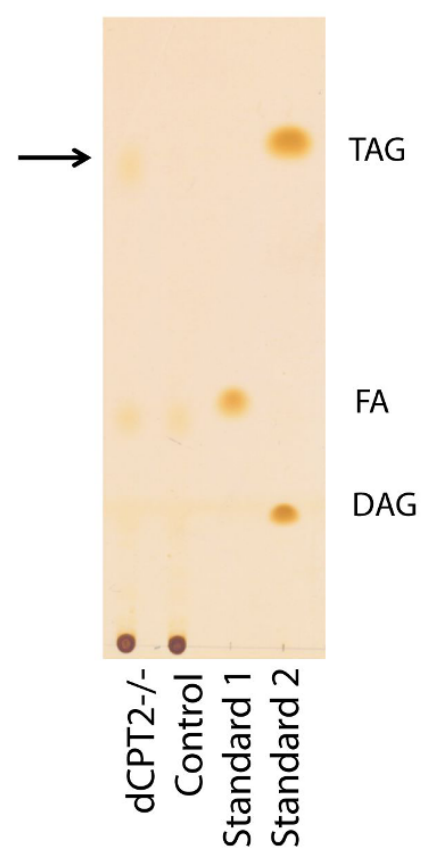

d
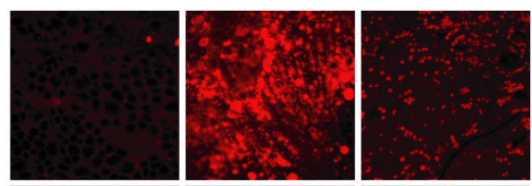

Brain
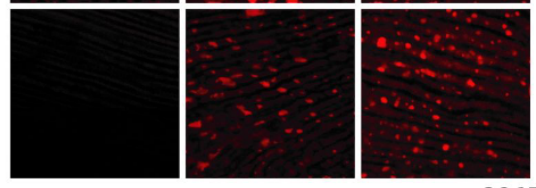

Muscle
Brain

uscle

Muscle

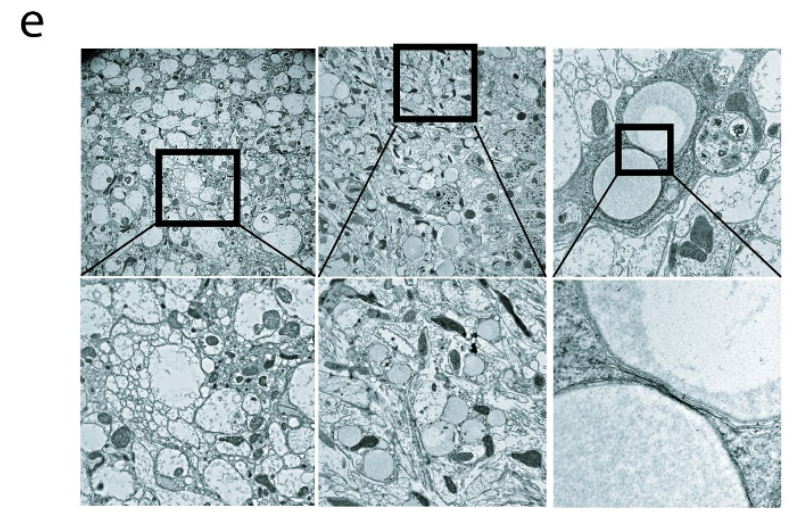

Control

\section{dCPT2-/-}

Figure 3 Lipid composition of $d C P T 2-/-$ flies and TAG accumulation in $d C P T 2-/-$ brains. (A) Protein, phospholipid (PL) and triacylglyceride (TAG) content of 1 week old adult female flies of the indicated genotype after the indicated hours of starvation ( $\mathrm{n}=3$ experiments). (B) Phospholipid $(\mathrm{PL})$ and triacylglyceride (TAG) content of 1 week old female adult brains of the indicated phenotype $(\mathrm{n}=3$ experiments, $* * * \mathrm{p}<0.001)$. $(\mathrm{C})$ Thin layer chromatography of lipid extracts of $d C P T 2-/-$ or wild type brains, fatty acid (FA; oleic acid), diacylglyceride (DAG; 1,2-diolein) and triacylglyceride (TAG, triolein) as standards. (D) Confocal micrograph of adult brains or flight muscle of the indicated genotype, stained with neutral lipid staining (red). (E) Electron micrograph of wild type or $d C P T 2-/-$ brain showing accumulation of TAG in $d C P T 2-/-$ brain marked with arrows. 
a

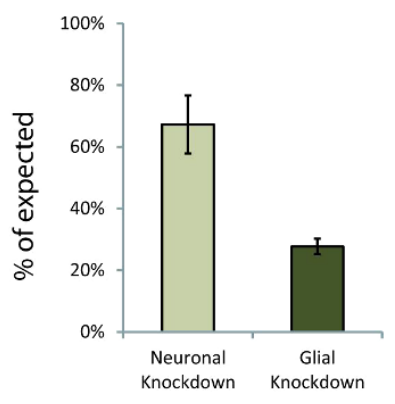

b

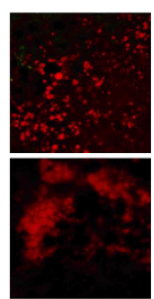

NLS

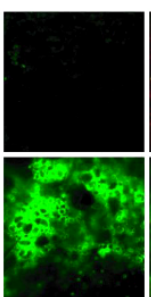

GFP

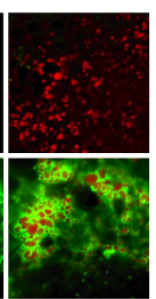

Merge
C

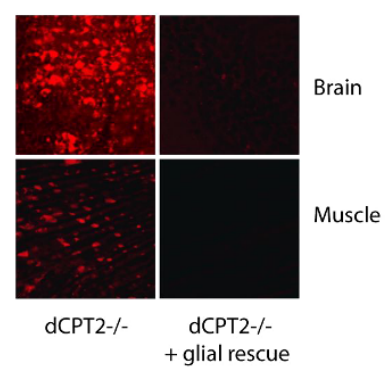

e
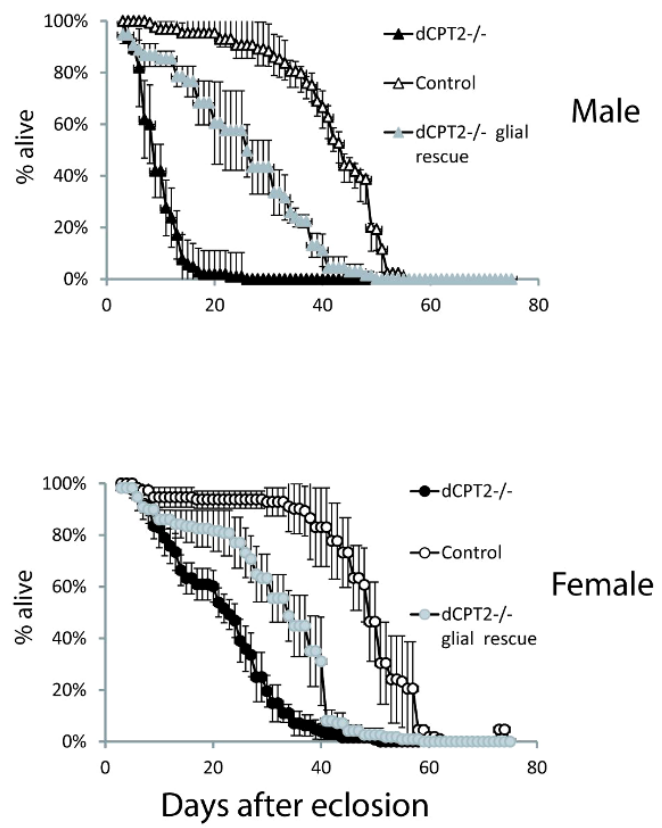

h

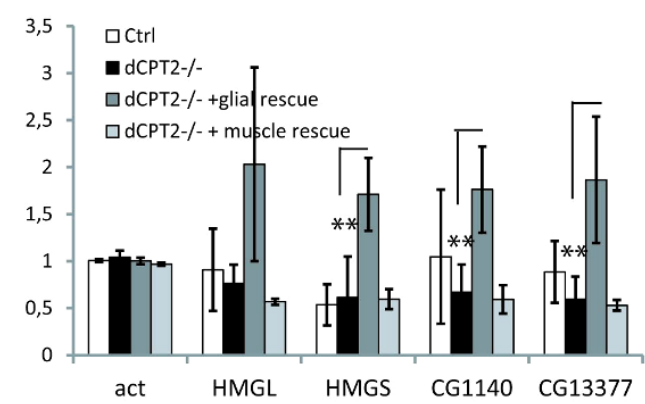

Figure 4 TAG localization in glial lipid droplets and contribution to organismal lipid metabolism. (A) Relative expression levels of dCPT2 mRNA in adult brain after specific knockdown in neurons (Nsyb-Gal4) or glia (Repo-Gal4) ( $\mathrm{n}=3$ experiments). (B) Confocal micrograph of adult $d C P T 2-/-$ brains expressing LSD2-GFP (in green) in neurons (Nsyb-Gal4) (upper row) or glia (Repo-Gal4) (lower row) and stained with neutral lipid staining (NLS) (red). (C) Confocal micrograph of adult brains and muscle from $d C P T 2-/-$ or $d C P T 2-/-$ with glial dCPT2 rescue (lower) flies stained with neutral lipid staining (red). (D) Eclosion viability of $d C P T 2-/-$ flies or $d C P T 2-/-$ flies with dCPT2 rescue in glia (Repo-Gal4), muscle (Mef2-Gal4), fat body $(C g-G a l 4)$ or neurons (Nsyb-Gal4) $\left(\mathrm{n}=3\right.$ experiments, $\left.{ }^{* * *} \mathrm{p}<0.001\right)$. (E) Lifespan of male or female wild type or $d C P T 2^{-/-}$adults with or without glial rescue with $\mathrm{rCPT} 2$, in days $+/-$ standard error of the means $(\mathrm{n}>=57$ flies). (F) Eclosion viability of dCPT2 $-/-$ flies or dCPT2 $-/-$ flies with dCPT2 rescue with the indicated glial subtype driver $(n=4$ experiments, $* * * p<0.001)$. (G) qPCR results for the indicated transcripts involved in different metabolic pathways, from RNA extracted from heads of the indicated genotypes with or without $24 \mathrm{~h}$ starvation (ratio starved/nonstarved) ( $\mathrm{n}=3$ experiments, $* * * \mathrm{p}<0.01)$. (H) $\mathrm{qPCR}$ results for the indicated transcripts involved in ketone body formation, from RNA extracted from heads of the indicated genotypes with or without $24 \mathrm{~h}$ starvation (ratio starved/nonstarved) $\left(\mathrm{n}=3\right.$ experiments, ${ }^{*} \mathrm{p}<0.05$ ). 
To analyze which metabolic changes underlie glial $d C P T 2^{-/-}$function, we compared expression levels of different genes involved in $\beta$-oxidation, lipogenesis and gluconeogenesis in heads of starved or non-starved adult wild type, $d C P T 2^{-/-}, d C P T 2^{-/-}$with glial dCPT2 rescue, and $d C P T 2^{-/-}$with muscular $\mathrm{dCPT} 2$ rescue flies. We found that HNF4, the master regulator of the $\beta$-oxidation transcriptional program ${ }^{17}$ was up regulated 1.6 fold in heads of $d C P T 2^{-/-}$glial rescue flies $(\mathrm{p}=0.001)$, but down regulated in heads of wild type (Fig. $4 \mathrm{~g}$ ), $d C P T 2^{-/-}$, or $d C P T 2^{-/-}$flies with muscular rescue, suggesting that the rescued glial cells perform $\beta$-oxidation. In contrast, the glial rescue flies did not induce lipogenesis, since SREBP levels remained constant, and gluconeogenesis was blocked, as indicated by the increase in PEPK levels $(\mathrm{p}=0.006)$.

How would $\beta$-oxidation restricted to glia provide energy to other cells such as neurons or muscle? This could happen in a manner similar to ketone body production in the mammalian liver, where this pathway is up regulated under starvation conditions. Thus we tested the brain for expressional changes of enzymes involved in ketone body generation. Strikingly, all four different enzymes involved in ketone body formation (HMGL, HMGS, CG1140 and CG13377) were up regulated 1.7 to 2.0 fold in $d C P T 2-/-$ flies with glial rescue ( $\mathrm{p}=0.02$ to 0.03 ), but not in wild type, $d C P T 2^{-/-}$or muscular rescue flies (Fig. $4 \mathrm{~h}$ ). This suggests that glial cells can provide energy to the organism by generation and export of ketone bodies derived from $\beta$-oxidation.

\section{Discussion}

The brain is a metabolically highly active tissue and more sensitive to nutrient and oxygen deprivation than any other organ. In fact, irreversible damage occurs already after minutes of hypoglycemia or ischemia ${ }^{18}$, while other organs survive for hours. Yet, a central dogma in biology is that $\beta$-oxidation of long-chain fatty acids (LCFA), an important source of ATP provision under starvation conditions, is completely absent from brain. Triacylglycerides (TAG) from which LCFA arise are also thought to be absent from this organ.

However, a number of observations challenge the dogma. First, the enzymes involved in mitochondrial $\beta$-oxidation are expressed in adult brain ${ }^{9}$. Second, individuals affected by a mutation in CPT2, an enzyme required for $\beta$-oxidation, have a morphological brain phenotype $^{7}$. Third, glial cells such as astrocytes are known to have a potential to provide metabolites such as lactate to neurons ${ }^{19}$. Fourth, neurons and glia are known to be able to store TAG under certain in vitro conditions ${ }^{20}$.

These reasons prompted us to investigate the potential of the brain to perform $\beta$-oxidation. We chose to study this under complete absence of $\beta$-oxidation in the whole organism, assuming that this might reveal $\beta$-oxidation induction deficits in the brain. We used the fruit fly as an animal model that is genetically tractable and created $\mathrm{CPT} 2^{-1-}$ flies. Those flies showed a phenotype reminiscent of human CPT2 deficiency. They die shortly after birth; the survivors have a reduced lifespan and are hypersensitive to starvation.

In support of our predictions, we could now demonstrate that CPT2 is expressed in the brain, mostly in glia. CPT2 $2^{-1-}$ but not wild type brains accumulated large amounts of TAG within lipid droplets in vivo, a phenomenon that has been observed only in vitro so far ${ }^{20}$. Detailed analysis demonstrated specific localization in glial cells, supporting their role as nurturing cells. To test the functional importance of glial cells in $\beta$-oxidation, we generated $\mathrm{CPT}^{-/-}$ animals in which CPT2 was rescued in glial cells only. This had both cell autonomous and non-autonomous effects. Autonomously, glial TAG accumulation was completely abolished, indicating that LCFA are degraded in glia and that glial cells are indeed capable of performing $\beta$-oxidation. Non-autonomously, organismal lethality and TAG accumulation in muscle is reversed. This could be mediated by an effect on the brain directly, causing improved brain function. Alternatively, glial cells could release energy metabolites such as ketone bodies back into the hemolymph for systemic use ${ }^{21}$. In support of the latter possibility, we found that enzymes involved in ketone body formation are up regulated in starving brains expressing CPT2 only in glia. This result is in turn consistent with early work showing that astrocytes in culture have the potential to produce ketone bodies from fatty acids ${ }^{22}$, altogether suggestive that the adult brain, under special circumstances like starvation, may act as a coordinator of organismal energy metabolism ${ }^{23}$.

Interestingly, $\mathrm{CPT} 2^{-1-}$ flies are not obese. This suggests that $\beta$-oxidation is not rate limiting for organism-wide energy storage in vivo and therefore not a potential target for the treatment of obesity, a condition associated with increased risk for age-related diseases and associated with a shortened lifespan ${ }^{24}$. Alternative mechanisms must exist that regulate the balance between organismal energy uptake and usage.

Our data are consistent with the following model of $\beta$-oxidation: (A) In wild type animals $\beta$-oxidation occurs in different tissues: TAG do not accumulate and enough energy is provided for survival. (B) When $\beta$-oxidation is ubiquitously impaired (such as in hereditary diseases), not enough energy can be provided leading to organismal dysfunction and death. In these situations TAG accumulate in the brain. (C) Allowing the animals with reduced (blocked) $\beta$-oxidation to perform $\beta$-oxidation specifically in glia results in energy production from LCFA $\beta$-oxidation and the export of ketone bodies to other tissues. This reverses TAG accumulation and prevents death. In support, a number of publications have reported that fatty acid oxidation/ketone body formation play a protective role.

The brain is a privileged organ in terms of substrate use for cellular energy production ${ }^{25}$. However, the brain is not completely but only relatively different from body cells, because in case of absolute need it is also capable of catabolizing LCFA for its own and even for systemic needs. This becomes relevant in ageing or obese organisms, where the substrate restriction might be increasingly loosened and $\beta$-oxidation less repressed ${ }^{26}$, as indicated by increasing expression of $\beta$-oxidation genes in ageing brains ${ }^{27,28}$. This might contribute to ageing phenotypes of the brain and increasing prevalence of agerelated neurodegenerative diseases such as Alzheimer or Parkinson disease.

\section{Methods}

Stocks. $P(G S V 7) G S 21694$, CPT1 EY20655/CyO, Df(3l)BSC428, Df(3L)BSC671 were received from Bloomington stock center. UAS $>$ LSD2-GFP was received from R. Kühnlein (MPI Göttingen); Repo-Gal4, Mef2-Gal4, Cg-Gal4, Nsyb-Gal4, NP 2222, Nrv2-Gal4, NP6520 and Eaat-Gal4 were received from M. Koch (VIB, Leuven).

Flies were raised on standard cornmeal molasses fly food ${ }^{29}$ and kept at $25 \mathrm{C}$ in a $12 \mathrm{~h}$ light/dark cycle.

$d C P T 2^{\delta 18}$ and $d C P T 2^{\delta 3}$ flies were generated by imprecise excision of $P(G S V 7) G S 21694$, located 92 bp upstream from the transcriptional start site of $C G 2107 . d C P T 2^{\delta 18}$ is a 456 bp deletion of CG2107 exon $1 . d C P T 2^{\delta 3}$ is a $3224 \mathrm{bp}$ deletion of the $5^{\prime}$ UTR and the first 505 amino acids of the CG2107 ORF. $d C P T 2^{\mathrm{Rev}}$ is a complete revertant and was used as control line. For targeted knockdown of dCPT2, UAS-line CG2107R2 from the NIG stock center was driven by Nsyb-Gal4 or Repo-Gal4.

Rescue constructs. Rat CPT2 cDNA was cloned into the EcoRI site of pUAST and checked for orientation. The H371A mutation was generated by site directed mutagenesis and the cDNA was recloned into pUAST.

cDNA was cloned into $\mathrm{pH}$-Pelican with the mammalian Hsp70 promoter and UAS binding sites. All constructs were verified by sequencing the complete insert and introduced into the germline by standard P-element mediated transformation.

dCPT2-GFP knock-in. The knock-in of GFP has been performed by the fosmid tagging method of Ejsmont et al. 2009 ${ }^{30}$. In brief, fosmid pFlyFos 015048, a clone from a genomic fosmid library containing an AttB sequence recognized by $\varphi \mathrm{C} 31$ integrase, the eye promoter-driven dominant selectable marker dsRed for isolation of transformants, and a $40 \mathrm{~kb}$ genomic sequence flanking $d C P T 2$ was used to C-terminally tag dCPT2 with super folder GFP by recombineering. The modified fosmid was verified by sequencing the tag-fosmid junction and injected into the germline of Drosophila melanogaster AttP2 flies. Revertant flies were selected with the help of dsRed driven in the adult eye. 
Lipid droplet staining. Adult brains were dissected in ice-cold PBS, fixed in $4 \%$ paraformaldehyde for $15 \mathrm{~min}$, washed $3 \times$ in PBS. Neutral Lipid Stain (HCS LipidTOX red neutral lipid stain, Molecular Probes, Eugene, Oregon) was added 1: 1000 in PBS for 30 min light protected at room temperature, brains were $3 \times$ washed, mounted in PBS and analyzed directly in a Biorad Radiance confocal microscope at $594 \mathrm{~nm}$.

qPCR. RNA was extracted from adult fly brains using Trizol. cDNA was synthesized with RevertAid First Strand cDNA Synthesis Kit using random primers. Quantitative PCR was performed with Light Cycler and SYBR green according to the manufacturer's protocol (Roche, Basel, Switzerland). Primers were designed to span an intron. Relative expression levels were quantified using the $2^{-\Delta \Delta \mathrm{Ct}}$ method.

act5c AGTCCGGCCCCTCCATT, CTGATCCTCTTGCCCAGACAA,

HNF4 CTGTCCAGATCCCCTTGTGT; GGCAGGATGAGCAGAATCTC,

SREBP AAGCTGAACAAGTCCGCAGT; CGCTTCTTGGATGCTCTACC,

PEPK GTGCCATCAACCCAGAGAAT; GCCCAACCAGTCAGTGATTT, ACC CG11198 TAACAACGGAGTCACCCACA,

CAGGTCACAACCGATGTACG,

HMG-CoA lyase TCAACCAATTGTCCGAAACA,

AGCACTCTCGAATCCCTTCA,

HMG-CoA synthase TGGTGTCTCTGTTGATTAGCG,

CTGATCGAGTACATGGAAGCAG, CG1140 CCTCATACTTTGGCTCCGATG

CCACCCATTCCCTTAACCAG

CG13377 AGTGTGGTCGCTTTGGATAC, CCGCTCGATCACATGAAGAG,

Lethality. Homozygous larvae were collected and added to a new food vial at non-crowded density. The percentage of eclosed flies was assessed.

Elextron microscopy. Adult brains were fixed in $2.5 \%$ glutaraldehyde and $2 \%$ paraformaldehyde in $0.1 \mathrm{M} \mathrm{Na}$-cacodylate buffer $\mathrm{pH} 7.4$ for 2 hours at room temperature and washed in $0.1 \mathrm{M} \mathrm{Na}$-cacodylate buffer. Then, they were post-fixed in $1 \%$ osmiumtetroxide $/ \mathrm{H}_{2} \mathrm{O}$ for 2 hours, dehydrated in a graded ethanol series and stained in uranyl acetate/70\% ethanol. Finally, the samples were infiltrated with $1: 1$ mixture of epoxy resin (Agar100) : propylene oxide for $60 \mathrm{~min}$ at $4{ }^{\circ} \mathrm{C}$ and left overnight in 2:1 mixture agar: propylene oxide, embedded and polymerized for 2 days in $100 \%$ agar. Finally, 50-60 nm sections were cut with a Leica UCT ultramicrotome and post-stained with uranyl acetate for $10 \mathrm{~min}$ and lead citrate for $3.5 \mathrm{~min}$. Brains were examined and photographed using a transmission electron microscope JEOL JEM-1400, equipped with a $2 \mathrm{k} \times 2 \mathrm{k}$ Veleta (Olympus SIS, Tokyo, Japan) camera.

Western blot. Flies were killed by freezing, homogenized with 10 strokes of an Eppendorf pestle in a buffer containing 1\% Triton X 100, and spun for $10 \mathrm{~min}$ at maximum speed in a table top centrifuge at $4 \mathrm{C}$. The supernatant was diluted to $2 \mathrm{ug} /$ ul protein content, boiled in SDS sample buffer, loaded on a Tris gel, transferred to a nitrocellulose membrane, blocked in non-fat dry milk, and visualized with $1: 1000$ anti-CPT2 (H-300 from Santa Cruz), a rabbit polyclonal antibody raised against amino acids 51-350 of human CPT2, or 1:1000 anti-Histone 3 (\#9715 from Cell Signaling) as loading control.

Life span. Flies were collected after birth and kept at $25^{\circ} \mathrm{C}$ in a humidified incubator with 12 hour light/dark cycle on regular corn molasses fly food. The vials were changed 3 times per week. Dead flies were scored every day.

Starvation. One week old flies $(\mathrm{n}>=80)$ were kept on $1 \%$ agarose at $25^{\circ} \mathrm{C}$ in a humidified incubator with 12 hour light/dark cycle. Death was removed and scored every 8 hours

Weight. The weight of 50 pooled one week old adult flies was quantified in a tared $1 \mathrm{ml}$ Eppendorf tube on a balance $(\mathrm{n}>=8)$.

Mobility. The motor activity of adult flies was quantified using a DAM activity monitoring system (Trikinetics, Waltham, MA).

Body length. The length of 10 adult, one week old flies per genotype was quantified under a Leica stereomicroscope.

Lipid analysis. Lipids were extracted from flies or brains with a modified Bligh \& Dyer procedure $^{31}$ and the extracts analyzed for phospholipids ${ }^{32}$, triacylglycerides ${ }^{33}$, fatty acids (NEFA kit; Wako, with some modifications) or separated by thin layer chromatography (silica $60 \mathrm{G}$ plates; $0.25 \mathrm{~mm}$; hexane/diethylether/acetic acid (70/ $30 / 1, v / v)$ ), followed by transient visualization with iodine vapor, scraping of spots of interest and analysis. The non-extracted material was dissolved in 1\% SDS and analyzed for protein content using bicinchoninic acid ${ }^{34}$

Whole mount brain confocal imaging. Briefly, adult brains were hand dissected in icecold PBS, and fixed for $10 \mathrm{~min}$ in 4\% PFA, mounted in Vectashield on glass slides and subjected to confocal imaging in a Bio-Rad Radiance confocal microscope (Bio-Rad).

1. Van Veldhoven, P. P. Biochemistry and genetics of inherited disorders of peroxisomal fatty acid metabolism. J Lipid Res 51, 2863-95 (2010).
2. Bieber, L. L. Carnitine. Annu Rev Biochem 57, 261-83 (1988).

3. Bonnefont, J. P. et al. Carnitine palmitoyltransferase deficiencies. Mol Genet Metab 68, 424-40 (1999).

4. Wolfgang, M. J. et al. The brain-specific carnitine palmitoyltransferase-1c regulates energy homeostasis. Proc Natl Acad Sci U S A 103, 7282-7 (2006).

5. Hug, G., Bove, K. E. \& Soukup, S. Lethal neonatal multiorgan deficiency of carnitine palmitoyltransferase II. N Engl J Med 325, 1862-4 (1991)

6. Taroni, F. et al. Molecular characterization of inherited carnitine palmitoyltransferase II deficiency. Proc Natl Acad Sci U S A 89, 8429-33 (1992).

7. North, K. N., Hoppel, C. L., De Girolami, U., Kozakewich, H. P. \& Korson, M. S Lethal neonatal deficiency of carnitine palmitoyltransferase II associated with dysgenesis of the brain and kidneys. J Pediatr 127, 414-20 (1995).

8. Pierce, M. R., Pridjian, G., Morrison, S. \& Pickoff, A. S. Fatal carnitine palmitoyltransferase II deficiency in a newborn: new phenotypic features. Clin Pediatr (Phila) 38, 13-20 (1999).

9. Lavrentyev, E. N., Matta, S. G. \& Cook, G. A. Expression of three carnitine palmitoyltransferase-I isoforms in 10 regions of the rat brain during feeding, fasting, and diabetes. Biochem Biophys Res Commun 315, 174-8 (2004).

10. Clarke, D. \& Sokoloff, L. Circulation and Energy Metabolism of the Brain. in Basic Neurochemistry (eds. Agranoff, B., Albers, R. W., Fisher, S. K. \& Uhler, M. D.) (Lipincott-Raven, Philadelphia, 1999).

11. Nunnari, J. \& Suomalainen, A. Mitochondria: in sickness and in health. Cell 148, 1145-59 (2012).

12. Brown, N. F., Anderson, R. C., Caplan, S. L., Foster, D. W. \& McGarry, J. D. Catalytically important domains of rat carnitine palmitoyltransferase II as determined by site-directed mutagenesis and chemical modification. Evidence for a critical histidine residue. J Biol Chem 269, 19157-62 (1994).

13. Granneman, J. G., Moore, H. P., Mottillo, E. P. \& Zhu, Z. Functional interactions between Mldp (LSDP5) and Abhd5 in the control of intracellular lipid accumulation. J Biol Chem 284, 3049-57 (2009).

14. Gronke, S. et al. Control of fat storage by a Drosophila PAT domain protein. Curr Biol 13, 603-6 (2003).

15. Edwards, T. N. \& Meinertzhagen, I. A. The functional organisation of glia in the adult brain of Drosophila and other insects. Prog Neurobiol 90, 471-97 (2010).

16. Melom, J. E. \& Littleton, J. T. Mutation of a NCKX eliminates glial microdomain calcium oscillations and enhances seizure susceptibility. J Neurosci 33, 1169-78 (2013).

17. Palanker, L., Tennessen, J. M., Lam, G. \& Thummel, C. S. Drosophila HNF4 regulates lipid mobilization and beta-oxidation. Cell Metab 9, 228-39 (2009).

18. Auer, R. N., Olsson, Y. \& Siesjo, B. K. Hypoglycemic brain injury in the rat. Correlation of density of brain damage with the EEG isoelectric time: a quantitative study. Diabetes 33, 1090-8 (1984).

19. Magistretti, P. J. Neuron-glia metabolic coupling and plasticity. J Exp Biol 209, 2304-11 (2006).

20. Du, L. et al. Starving neurons show sex difference in autophagy. J Biol Chem 284, 2383-96 (2009).

21. Guzman, M. \& Blazquez, C. Is there an astrocyte-neuron ketone body shuttle? Trends Endocrinol Metab 12, 169-73 (2001).

22. Edmond, J., Robbins, R. A., Bergstrom, J. D., Cole, R. A. \& de Vellis, J. Capacity for substrate utilization in oxidative metabolism by neurons, astrocytes, and oligodendrocytes from developing brain in primary culture. J Neurosci Res 18, 551-61 (1987).

23. Rajan, A. \& Perrimon, N. Drosophila as a model for interorgan communication: lessons from studies on energy homeostasis. Dev Cell 21, 29-31 (2011).

24. Haslam, D. W. \& James, W. P. Obesity. Lancet 366, 1197-209 (2005).

25. Cheng, L. Y. et al. Anaplastic lymphoma kinase spares organ growth during nutrient restriction in Drosophila. Cell 146, 435-47 (2011).

26. Karmi, A. et al. Increased brain fatty acid uptake in metabolic syndrome. Diabetes 59, 2171-7 (2010).

27. Berchtold, N. C. et al. Gene expression changes in the course of normal brain aging are sexually dimorphic. Proc Natl Acad Sci U S A 105, 15605-10 (2008).

28. Loerch, P. M. et al. Evolution of the aging brain transcriptome and synaptic regulation. PLoS One 3, e3329 (2008).

29. Schulz, J. G. et al. Drosophila syndecan regulates tracheal cell migration by stabilizing Robo levels. EMBO Rep 12, 1039-46 (2011).

30. Ejsmont, R. K., Sarov, M., Winkler, S., Lipinski, K. A. \& Tomancak, P. A toolkit for high-throughput, cross-species gene engineering in Drosophila. Nat Methods 6 , 435-7 (2009).

31. Van Veldhoven, P. P. \& Bell, R. M. Effect of harvesting methods, growth conditions and growth phase on diacylglycerol levels in cultured human adherent cells. Biochim Biophys Acta 959, 185-96 (1988).

32. Van Veldhoven, P. P. \& Mannaerts, G. P. Inorganic and organic phosphate measurements in the nanomolar range. Anal Biochem 161, 45-8 (1987).

33. Van Veldhoven, P. P., Swinnen, J. V., Esquenet, M. \& Verhoeven, G. Lipase-based quantitation of triacylglycerols in cellular lipid extracts: requirement for presence of detergent and prior separation by thin-layer chromatography. Lipids 32, 1297-300 (1997). 
34. Smith, P. K. et al. Measurement of protein using bicinchoninic acid. Anal Biochem 150, 76-85 (1985)

\section{Acknowledgments}

We thank Pedro F. Marrero (U Barcelona) for cDNA rCPT2, P. Tomancaz for pFlyFos 015048, M. Koch (VIB, Leuven), Bloomington and NIG stock centre, and R. Kühnlein for stocks, T. Holmes for help with the DAM system, Pieter Baatsen (EM-facility EMoNe, VIB Bio Imaging Core/KU Leuven) for help with electron microscopy, R. Ejsmont for help with dCPT2 GFP knock-in, Guy Froyen (KU Leuven) for help with qPCR, Evelyne de Schryver (KU Leuven) for help with lipid analysis and fatty acid oxidation experiments. This work was partially supported by the Flanders Fund for Scientific Research (FWO G 0.666.10N), NEUROBRAINNET IAP 7/16, Flemish Government Methusalem Grant, Spanish Ministry of Science (SAF2010-14906) and Innovation Ingenio-Consolider (CSD2010-00045) and Spanish Ministry of Economy and Competitiveness (SAF2013-45392).

\section{Author contributions}

J.G.S., A.L., L.V.H., A.G., S.V., J.B. and P.P.V.V. performed the experiments, J.G.S. and C.G.D. supervised the project.

\section{Additional information}

Supplementary information accompanies this paper at http://www.nature.com/ scientificreports

Competing financial interests: The authors declare no competing financial interests.

How to cite this article: Schulz, J.G. et al. Glial $\beta$-Oxidation regulates Drosophila Energy Metabolism. Sci. Rep. 5, 7805; DOI:10.1038/srep07805 (2015).

This work is licensed under a Creative Commons Attribution-NonCommercialShareAlike 4.0 International License. The images or other third party material in this article are included in the article's Creative Commons license, unless indicated otherwise in the credit line; if the material is not included under the Creative Commons license, users will need to obtain permission from the license holder in order to reproduce the material. To view a copy of this license, visit http:// creativecommons.org/licenses/by-nc-sa/4.0/ 